Revista Oficial del Poder Judicial

ÓRGANO DE INVESTIGACIÓN DE LA CORTE SUPREMA DE JUSTICIA DE LA REPÚBLICA DEL PERÚ

Vol. 13, n. ${ }^{\circ} 16$, julio-diciembre, 2021, 77-99

ISSN: 1997-6682 (Impreso)

ISSN: 2663-9130 (En Línea)

DOI: 10.35292/ropj.u13i16.360

\title{
El conflicto entre los criterios de valoración probatoria y la construcción de un proceso penal con perspectiva de género
}

The conflict between evidentiary assessment criteria and the construction of a criminal process with a gender perspective $\infty$ (우

LUCIANA NATALIA UGARRIZA LANDAVERY

Corte Superior de Justicia de La Libertad

(Trujillo, Perú)

Contacto: lugarrizal@pj.gob.pe

https://orcid.org/0000-0002-0652-5153

\section{RESUMEN}

En el presente artículo se analizan los problemas que enfrenta en la actualidad el proceso penal en cuanto a la declaración del testigovíctima en delitos basados en género, producto de un desenfrenado populismo e insatisfacción ciudadana. Resulta necesario evitar cualquier sesgo de género por el cual se pueda arribar a resoluciones judiciales irracionales, ello sin disminuir las garantías procesales, especialmente las que asisten a los investigados. 
Palabras clave: prueba; perspectiva de género; presunción de inocencia; criterios de valoración; violencia de género; condición de vulnerabilidad.

\section{ABSTRACT}

This article analyzes the problems currently facing the criminal process in terms of the testimony of the witness-victim in gender-based crimes, a product of rampant populism and public dissatisfaction. It is necessary to avoid any gender prejudice that could lead to irrational judicial decisions, without diminishing the procedural guarantees, especially those of the investigated.

Key words: evidence; gender perspective; presumption of innocence; assessment criteria; gender violence; condition of vulnerability.

Recibido: 10/05/2021 Aceptado: 01/09/2021

\section{INTRODUCCIÓN}

No es casualidad que el fenómeno de la violencia tenga mayor incidencia en mujeres, quienes a lo largo de la historia se han visto desfavorecidas en distintos ámbitos, como los sociales, los laborales, los culturales, etc., fruto de la representación constitutiva y legitimadora del orden social, la cual asigna roles específicos a hombres y mujeres. A la vista y análisis de este panorama de discriminación, surgen distintos movimientos a partir del siglo XIX, que buscan remover la violencia por razones de género (de allí que se asocie más con las mujeres que con los hombres), pero ¿qué entendemos por este término?

Es necesario empezar definiendo al género, que no es más que el conjunto de estereotipos sociales y culturales asociados a un determinado sexo (esto quiere decir que la persona no sufre violencia 
por las características físicas del sexo sino por dichos estereotipos asociados a este último). Ahora, en aras de contrarrestar este escenario, se buscó introducir una herramienta que tenga presente esta situación histórica y que propugne la eliminación de prejuicios basados en la inferioridad o la superioridad: la perspectiva de género.

Así, el Estado ha avanzado con el propósito de construir una sociedad cada vez más igualitaria mediante la ratificación de tratados, instrumentos internacionales y emisión de leyes de tipo sancionador que buscan erradicar todo tipo de violencia contra las mujeres, que en la actualidad son identificadas como el grupo en situación de vulnerabilidad más numeroso y con mayor índice de atentados contra su integridad, por lo que la Corte Suprema reconoce que se debe administrar justicia con «perspectiva de género» a fin de evitar la impunidad y seguir la tendencia social que clama por el castigo.

Actualmente, la protección solo de mujeres frente a la violencia de género se sigue valorando por amplios sectores de la opinión pública como un privilegio injustificado, por lo que muchas veces - o casi siempre - se «sitúa a los jueces en una posición complicada que podría acrecentar la posibilidad de arribar a soluciones sub óptimas, que atentan con defenestrar el proceso penal, en perjuicio de las personas imputadas» (Mora, 2020, p. 67). Esto tiene que ver directamente con la presunción de inocencia, pues para poder desvirtuarla, se exige una rigurosa valoración probatoria, más aún en delitos con penas tan elevadas en donde el estándar de convicción que logre el juez para emitir una sentencia condenatoria efectiva debería ser mayor.

En las siguientes líneas se pretende analizar la necesidad de precisar algunos criterios de valoración probatoria, considerando que en delitos de esta índole, al ser mayoritariamente cometidos en la clandestinidad, solo se cuenta con el testimonio de la víctima. 
Teniendo claro el panorama, cabe preguntarnos qué debe y puede hacer el derecho penal en materia de delitos relacionados con violencia de género - específicamente respecto a los criterios de valoración probatoria que se deberían tener en cuanto a la declaración única de la víctima-, así como qué puede aportar la perspectiva de género al mismo problema sin colisionar con los principios de primer orden que fundan el Estado de derecho.

\section{PRESUNCIÓN DE INOCENCIA}

De acuerdo con la sociología, tenemos una tendencia a creer todo lo que nos dicen, y no solamente creer, sino a compartirlo, como mecanismo para crear lazos entre la sociedad. Sin embargo, lo más curioso es que solemos creer más en lo negativo que en lo positivo, la gran pregunta es ¿por qué?, pues simple y sencillamente por algo que condiciona al ser humano y a cualquier otra especie: instinto de supervivencia. Supongamos que nos cuentan que Pedro es un asesino, inmediatamente nos veremos en la necesidad de creer dicha proposición con el fin de alejarnos de Pedro, de no tener ningún tipo de contacto con él, porque nos puede hacer daño (Nieva, 2016b).

Siguiendo a Díaz (2019):

Atrás quedaron los años en los cuales la inquisición, el castigo y la tortura eran indispensables para poder hallar culpables e imponer penas, con la revolución se dio cuenta de la necesidad de presumir la inocencia de la persona. [...] este es un derecho reconocido ampliamente, al menos, en el mundo occidental (p. 20).

Entrar al análisis partiendo de la historia, sociología y psicología, nos permite entender el motivo por el cual nace la presunción de inocencia. El profesor español Nieva Fenoll (2016a) sostiene que la presunción de inocencia tiende a reequilibrar esa situación precaria que tiene el reo en el proceso penal; sin embargo, no es 
como cualquier otra parte, porque es aquel al que todo el mundo ve como culpable desde el primer momento en el que roza el banquillo.

Lo cierto es que el simple hecho de señalar a una persona como sospechosa, genera automáticamente un recelo social ante ese individuo. Es muy raro que alguien le tenga por inocente.

[...]

Ante esta realidad, no es extraño que con el objetivo de evitar las falsas acusaciones que generan siempre un perjuicio social notable, surgiera la idea de la presunción de inocencia. Con el fin de que la sociedad no fuera generando a través de rumores una verdad ficticia (Fenoll, 2016, pp. 5-6).

En palabras del autor nacional Castillo Alva (2020):

La presunción de inocencia, como un derecho procesal, se relaciona estrechamente con el respeto del derecho de defensa y, al mismo tiempo, promueve el respeto por el honor y la dignidad del acusado. En tales situaciones, la presunción de inocencia despliega una prevención frente a cualquier pronunciamiento injusto que pretenda establecer la culpabilidad de la persona (p. 204).

Nos encontramos, pues, ante una tendencia social que señala que todos los investigados son culpables, en un tiempo caracterizado por la sustitución del paradigma de la presunción de inocencia en el proceso penal, focalizando toda la atención en la víctima. Por ello es importante averiguar si la presunción de inocencia debe ser clave en materia probatoria o se necesita otra alternativa. En ese sentido, Ramírez (2020) lo llama «derecho penal identitario», que vendría ser una nueva modalidad del derecho penal en donde lo que se protege no es a ciudadanos sino a colectivos, y si bien las mujeres abarcan la mitad de la población, estaríamos en retroceso en cuanto a la naturaleza del proceso penal. En el siguiente punto analizaremos algunas consideraciones sobre la violencia basada en género, a fin de entender la tendencia social aludida. 


\section{PANORAMA EVALUATIVO SOBRE LA VIOLENCIA BASADA EN GÉNERO}

El profesor español Lousada Arochena (2020) señala que

La funcionalidad más profunda de la violencia de género en un sistema patriarcal es que a través de su ejercicio se actualizan en la realidad de la vida los estereotipos de dominación asignados al hombre y los estereotipos de sumisión asignados a la mujer: en cualquier forma de violencia de género ejercida por un hombre sobre una mujer, aquel está adoptando una posición dominante, y esta debe ser sometida, colocándose hombre y mujer en la posición asignada socioculturalmente (pp. 59-60).

En palabras del costarricence Mora (2020), a la violencia de género debe atribuírsele un concepto con sentido social, pues «el basamento de este tipo de violencia es un estereotipo de género, asociado firmemente con un modelo normativo violento de la masculinidad [...] solamente esta noción es propicia para ofrecer un norte adecuado en el abordaje del fenómeno» (p. 77).

En el Acuerdo Plenario del X Pleno Jurisdiccional de las Salas Penales Permanente y Transitorias se sostiene que

Es necesaria la reacción contra la violencia de género que afecta a la mujer (por el solo hecho de serlo) que existe como fenómeno social (que tiene su origen en una situación de discriminación, desigualdad y de relaciones de poder entre el hombre y la mujer), y una de las medidas necesarias es su tipificación como delito como línea de acción para evitar su comisión (en torno a un paradigma de prevención general y especial) (p. 33).

En esa misma idea, Bendezú Barnuevo (2017) señala que la denominada violencia de género hace referencia a la violencia ejercida por algunos varones contra mujeres, a causa de las relaciones de poder, dominio y posesión que estos han ostentado históricamente aquellos sobre aquellas especialmente en el ámbito de la pareja (p. 38). 
Igualmente, Reátegui Sánchez y Reátegui Lozano (2017) señalan que

La violencia es de género, porque recae sustancialmente sobre la mujer, la violencia es el poder y el poder genera sumisión, daño, sufrimiento, imposición de una voluntad, dominación y sometimiento, pues la violencia presupone, por lo general, posiciones diferenciadas, relaciones asimétricas y desiguales de poder, la violencia de género implica todo esto y mucho más, cuya hiperincriminación se justifica, precisamente, porque germina, se desarrolla y ataca en un contexto específico: el contexto de género (p. 37).

Contra tales posiciones adoptadas, Castillo Aparicio (2019) afirma lo siguiente:

El fundamento de que la violencia de género es solo violencia contra las mujeres es erróneo, pues si bien la violencia contra las mujeres es una de las formas de violencia de género, ello no excluye que hay otras formas de violencia de género como las que sufren las personas de diversidad sexual. [...] Por tanto la violencia de género no se ejerce únicamente sobre mujeres, aunque cuantitativamente pueda ser el sector más afectado por la misma (p. 37).

Particularmente, adopto la última postura, que tiene una noción más tendencial. Sin embargo, no cabe duda de que la sociedad peruana, como muchas otras, se ha moldeado bajo ciertos parámetros que se encuentran motivados por la imposición del cumplimiento de determinados estereotipos a cada sexo, en especial a las mujeres, lo cual se refleja en los elevados índices de criminalidad hacia estas. Tal y como lo señala Mora Sánchez (2020):

Cotidianamente existe un entorno hostil para las mujeres, difuminándose la percepción real de la violencia en ese ambiente normalizador. Así, las burlas ofensivas pasan por folklor popular, los actos de acoso se mimetizan como simples halagos, el sufrimiento se trivializa y los golpes y el abuso se justifican (p. 66). 


\section{CRITERIOS DE VALORACIÓN EN LA DECLARACIÓN DE LA VÍCTIMA-TESTIGO CON PERSPECTIVA DE GÉNERO}

En materia de análisis de la prueba en el proceso penal, surge un tema bastante polémico en cuanto al testimonio de la víctima o testigo en delitos basados en violencia de género. Por ello, el objetivo principal de este trabajo es analizar hasta qué punto podemos exigir a los órganos jurisdiccionales que administren justicia con perspectiva de género, y si es necesario precisar ciertos límites respecto a la valoración de la declaración única de la víctima, que no genere una convicción en el juez a causa de una mera intuición, sino producto de una actividad racional.

Es sabido que el testimonio es un medio de prueba de suma importancia en todos los procesos, pero en particular se suele utilizar como prueba de cargo en los procesos por delitos de género, tomando como base el hecho de que la víctima es el principal (y único) testigo. Sin embargo, si lo que buscamos es lograr una verdadera justicia, no podría afirmarse que en todos los asuntos en materia de violencia de género deba creerse a la presunta víctima, por lo que nos preguntamos: ¿la perspectiva de género serviría para justificar una acusación ante el supuesto límite de escasez de prueba (no corroboración de la declaración)? Ante tal disyuntiva probatoria, se han adoptado las siguientes decisiones estatales:

\subsection{Acuerdo Plenario n. ${ }^{0}$ 02-2005}

A través de este acuerdo plenario se establecieron como reglas de valoración de las declaraciones de víctimas y testigos, las siguientes:

a) «Ausencia de incredibilidad subjetiva. Es decir, que no existan relaciones entre agraviado e imputado basadas en el odio, resentimientos, enemistad u otras que puedan incidir en la parcialidad de la deposición, que por ende le nieguen aptitud para generar certeza» (fundamento jurídico 10). 
En este primer punto, es importante que los jueces determinen si la relación acusado-víctima denota algún móvil de odio, resentimiento, venganza, enemistad u otro, puesto que de ser así, se acrecentaría un estado de incertidumbre y sospecha respecto a los hechos denunciados. No obstante, también, aunque exista un móvil de por medio, no debería eliminarse automáticamente el valor de la declaración, pues con ello estaríamos afirmando que sería imposible que mi enemigo pueda ser mi ofensor, esto es, una emoción como el odio de por medio no convierte automáticamente en falsa una declaración. Esto, según Pizarro (2020):

implica que la concurrencia de alguno de estos escenarios, es solamente una llamada de atención al juzgador para realizar un filtro cuidadoso de la declaración, no debiéndose descartar aquellas que, aun teniendo estas características, tienen solidez, firmeza y veracidad objetiva (p. 20).

En cuanto a ello, Nieva Fenoll (2010), citando a Manzanero, destaca respecto de la psicología del testimonio que ha puesto en evidencia la necesidad de valorar la credibilidad del testimonio y no del testigo (p. 220). Por ello, se ha señalado que debe «abolirse la costumbre de que los jueces valoren a los declarantes por su conducta durante la declaración [...] es ya hora de que la firmeza o vehemencia de quien hable deje de confundirse con su credibilidad» (p. 239).

Por este motivo, es más importante el contenido de la declaración que quien declara, pues de esta manera evitaremos muchos sesgos. Es así que el juez debe analizar si los móviles mencionados están relacionados con hechos anteriores al supuesto delictivo o no, ya que es entendible que toda persona afectada con esta clase de delitos genere una reacción contraria al agresor, un distanciamiento con él. 
b) «Verosimilitud, que no solo incide en la coherencia y solidez de la propia declaración, sino que debe estar rodeada de ciertas corroboraciones periféricas, de carácter objetivo que le doten de aptitud probatoria» (fundamento jurídico 10).

El profesor Higa (2019) apunta que

Si bien los actores serán una fuente de información, se debe investigar qué tan fiable o creíble puede ser la información que proporcionen [...] dado que esta se encontrará supeditada a los criterios que se deben evaluar para la admisión y valorar la declaración de una persona (p. 52).

Nieva (2010) precisa, por otro lado:

en cuanto a la coherencia del relato del testigo-víctima, no es una invariable señal de verosimilitud, pues incluso podría ser un indicador de todo lo contrario, en la medida que los testimonios falsos suelen presentarse de una manera continuamente estructurada y generalmente cronológica (p. 224).

La perspectiva de género además de aportar nuevas máximas de la experiencia para valorar este acuerdo plenario, nos permite adentrarnos en otro ámbito. Cuando vamos al contenido de la declaración de la víctima, nos encontramos con dos elementos fundamentales: coherencia en el relato y existencia de corroboraciones que confirman ese relato.

En cuanto a este criterio, es importante precisar cuándo hablamos de coherencia y solidez. El primer término es una propiedad de los textos bien formados, que hace posible que estos sean concebidos como entidades unitarias; así las diversas ideas secundarias aportan información relevante que permite llegar a la idea principal o tema. De este modo el lector puede hallar el significado global del texto. La coherencia alude a la existencia de una acción que resulte fácilmente identificable y que se 
encuentre asociada a un contexto que proporcione una explicación del comportamiento de las personas que intervienen. Aquí se visualiza el aporte de la perspectiva de género, pues un mismo relato puede valorarse como creíble o no creíble dependiendo de la sociedad en la cual vivamos. Respecto a la solidez, esta se refiere a que el relato tiene contundencia porque no es fantasioso, sino que se trata de un relato creíble o posible.

Coincidimos con el maestro Pizarro Guerrero (2019) en que «no es posible afirmar que la versión de la testigo-víctima debe coincidir en un cien por ciento con la versión de otras personas. Solo se exige que en sus extremos esenciales el relato sea consistente y coincidente» (p. 223). Sin embargo, consideramos que a la manifestación subjetiva de la víctima, debe añadírsele algún dato que apoye a dicha manifestación. Entre estos pueden estar las testimoniales, las lesiones en delitos que ordinariamente las producen, periciales, o incluso que su propio relato sea lo suficientemente creíble al proporcionar datos que no sean de conocimiento público, como características corporales del imputado, etc.

c) Persistencia en la incriminación. Es decir, que debe existir persistencia en sus afirmaciones en el curso del proceso. Esto es, tal y como lo plasma el profesor Pizarro (2019):

ausencia de modificaciones esenciales en las sucesivas declaraciones prestadas por la víctima sin contradecirse ni desdecirse: concreción en la declaración que ha de hacerse sin ambigüedades, generalidad o vaguedades [...] y coherencia o ausencia de contradicciones, manteniendo el relato la necesaria conexión lógica entre sus diversas partes (p. 225).

Cuando entra en vigencia el Acuerdo Plenario n. ${ }^{\circ}$ 02-2005, no se contaba con que se iba a hacer una recesión acrítica del contenido que se convertiría en una forma automática de valorar la prueba de modo que el juzgador, cuando concurren esos tres criterios, automáticamente otorga el valor de prueba de cargo a 
la declaración de la víctima o testigo, ignorando que se trata de simples directrices o guías, que han de ser tomadas en consideración para valorar.

Coincidimos con el profesor Talavera, citado por Pizarro (2019), cuando sostiene que

Aunque con bastante frecuencia se han venido considerando los referidos criterios valorativos como requisitos, no son tales, sino simples directrices o criterios para realizar una adecuada crítica del testimonio de la víctima y determinar si tiene aptitud o no para ser considerada como prueba de cargo (p. 245).

Sobre este punto, en la R. N. n. ${ }^{\circ}$ 2633-2017-San Martín, la Primera Sala Penal Transitoria absolvió al condenado incluso cuando existió persistencia en la incriminación, sostuvieron que esta por sí sola no va a generar en el juez la convicción ausente de toda duda razonable sobre la responsabilidad del imputado, pues un hecho falso también puede ser persistente en el tiempo, y tiene que compensarse con el análisis de los demás requisitos detallados ut supra.

Esto no significa que un testimonio que supere esos tres filtros debe ser considerado inculpatorio. Debemos precisar aquí que un testimonio que no lo hiciera tendría que ser desestimado como medio de prueba, mientras que, en caso contrario, resultará considerable y cabría pasar a confrontar dicha aportación con otro tipo de corroboraciones. Por tanto, dichos filtros tienen una función orientadora, a fin de obtener una mayor racionalidad en la valoración de la prueba.

\subsection{Recurso de nulidad n. ${ }^{398-2020}$ Lima Norte}

Este recurso señala en su considerando 3.4, que respecto a la valoración de la prueba, debe realizarze con perspectiva de género. Así, establece lo siguiente: 
c) Siguiendo los lineamientos de la Corte Interamericana de Derechos Humanos con relación a la obligación de valorar las pruebas con perspectiva de género: i) no se puede esperar la inexistencia de inconsistencias o imprecisiones en los testimonios de las víctimas, como cuestiona el impugnante, pues los hechos ocurrieron de forma rápida, según señalaron las agraviadas y el procesado; ii) tampoco puede exigirse a las tres personas que estuvieron en el lugar de los hechos un relato milimétrico sobre cómo fue, minuto a minuto, cada agresión o suceso; peor todavía considerando la diferencia de tiempo entre sus declaraciones, esto es, las primeras fueron recibidas el día de ocurrido el hecho o al día siguiente, y las últimas luego de haber transcurrido casi un año; iii) menos aún puede esperarse que siempre existan testigos o pruebas documentales de las agresiones físicas o psicológicas que sufren las víctimas.

En el caso de la violencia de género, a manera de ejemplo, la demora en la interposición de la denuncia puede ser considerada como una falta de veracidad, y es ahí donde entra a tallar la perspectiva de género, la cual nos permite visualizar que en contextos marcados por la dominación y el patriarcado, la persona que esté en una situación de inferioridad, puede generar sentimientos de miedo hacia el agresor y eso puede justificar la demora en la interposición de su denuncia.

En ese sentido, Ramírez (2020) afirma:

La perspectiva de género exige que el relato que realiza la mujer que narra haber sido víctima de actos violentos protagonizados por el hombre se evalúe eliminando estereotipos que tratan de universalizar como criterios de racionalidad simples máximas de experiencia machistas (v. gr. el estereotipo de «víctima ideal» que, tras sufrir el hecho, lo denuncia inmediatamente, mantiene siempre un relato idéntico de lo acontecido y se aísla socialmente; el estereotipo de mujer sexualmente disponible para cualquier 
hombre, inducido de datos con pretendido valor indiciario tales como su vestimenta, su estado de embriaguez o el lugar y hora de la noche en la que se encuentra [...] (p. 220).

No obstante, eso significaría, a manera de ejemplo, que siempre que exista una demora en la interposición de la denuncia ¿tenemos a un testigo/víctima creíble?, ¿o a un testigo al que no debamos creerle? Otros ejemplos claros son los casos en que la víctima se genera un reproche a sí misma por haber interpuesto una denuncia en contra de algún familiar o conocido, y llega a sentirse culpable del distanciamiento entre los miembros de su familia, o que producto de ello el ofensor dejará de otorgarle una pensión económica.

\subsection{Acuerdo Plenario n. ${ }^{\circ} 1-2011 / C J-116$}

Este recurso señala en su fundamento jurídico vigésimo tercero que

Se ha establecido anteriormente - con carácter de precedente vinculante - que al interior del proceso penal frente a dos o más declaraciones carentes de uniformidad o persistencia —en cuanto a los hechos incriminados - por parte de un mismo sujeto procesal: co-imputado, testigo víctima, testigo, es posible hacer prevalecer como confiable aquella con contenido de inculpación por sobre las otras de carácter exculpante. Dicho criterio encuentra particular y especial racionalidad precisamente en este ámbito de delitos sexuales en los que es común la existencia de una relación parental, de subordinación o de poder entre agente y víctima.

Existe armonía, pues, en estas resoluciones respecto a que se debe seguir resolviendo con perspectiva de género en casos en los que se cuente únicamente con la declaración del testigo/víctima. No podemos ignorar que aún vivimos en una sociedad patriarcal, donde ciertas conductas son, con mayor frecuencia, atribuibles a mujeres y otras a hombres. El principal aporte que puede hacer la 
perspectiva de género en los supuestos de testimonio único es en la fase de investigación, porque se puede indicar al fiscal qué elementos de corroboración han de recopilar para que cuando se celebre el juicio oral puedan confirmar la declaración, de manera que nos va a indicar cómo son las relaciones caracterizadas por situaciones de subordinación entre víctima y victimario.

En el plano del juicio oral, puede servir para valorar la prueba sin que medie ningún estereotipo de género, que esté vinculado a la atmósfera cultural del juez, es decir, los jueces evalúan los hechos e interpretan los datos de la realidad según cómo comprenden el mundo. La presencia de estereotipos en la percepción de las autoridades invisibiliza la situación de violencia existente y trae consecuencias trágicas, por lo que aplicando una perspectiva de género, esta situación se identificaría y se tomarían las medidas adecuadas de protección. Mantilla (2016), al respecto, sostiene que «si bien la derogación y/o modificación de las normas jurídicas son importantes, el real problema se encuentra en quienes tienen a cargo la interpretación y aplicación» (p. 122). Entonces, esta perspectiva interviene como elemento crítico para cuestionar de qué forma evalúan esa realidad.

\section{TESTIMONIO ÚNICO ANTE LA PRESUNCIÓN DE INOCENCIA}

En merced del concepto de perspectiva de género, que puede ser muy valioso, se está produciendo tanto una debilitación de las garantías del proceso como de la valoración probatoria. Es importante dejar en claro que el análisis de las resoluciones descritas no debe ser absoluto, es decir, no significa que la sola declaración de la víctima-testigo va a conllevar a una condena, pues sería incompatible con el principio de igualdad consagrado en nuestra constitución y con el principio de presunción de inocencia. 
La maestra Pérez Cepeda (2007) afirma:

La distorsión de la figura de la víctima dentro de los procesos penales ha provocado ( $y$ continúa haciéndolo) una severa dislocación del engranaje garantista consustancial al modelo punitivo requerido en un Estado de derecho. Se trata de la construcción de una posición parcial y poco favorable a la racionalidad, lo que determina una presión social sobre el Derecho penal que no solo tiende a ampliarlo, sino también que, este desequilibrio entre la víctima y el delincuente, se pone de manifiesto con una predisposición a negar derechos de estos últimos (p. 439).

Lo que no se puede pretender es que la coherencia del relato, enriquecida por los aportes de la perspectiva de género, satisfaga todas las exigencias que son necesarias para abastecer del peso probatorio con prueba de cargo a la declaración de la víctima/ testigo. Es necesaria la corroboración y presencia de actos externos que confirmen lo que el testigo ha manifestado, porque solamente atendiendo a la coherencia del relato, si este no se halla sujeto a un elemento externo, al final la decisión caerá en la subjetividad del juez y no se satisfacen las exigencias de la presunción de inocencia.

Además, debe señalarse que

la aparente sencillez o simplicidad procesal que puede denotar un caso penal $[\ldots]$ no exime que una acusación fiscal esté debidamente respaldada de cierto nivel probatorio que le dé fundabilidad. El formular acusación directa con indicios que solo alcanzan nivel de sospecha reveladora es una aventura a la cual el Ministerio Público se arriesga debido a que son esos los indicios que le servirán para sustentar su teoría del caso y obtener una futura condena, una empresa bastante difícil de lograr en esas condiciones (fundamento jurídico décimo primero). 
En mi opinión, la presunción de inocencia no se puede someter a examen, en caso contrario, estaríamos destruyendo su contendido como principio general. En muchos estados latinoamericanos, se han elaborado guías de enjuiciamiento con perspectiva de género, se han establecido reglas específicas que invitan a flexibilizar la valoración probatoria y la aplicación de criterios probatorios en delitos de violencia de género a fin de que se utilice la perspectiva de género; empero, lo único que están logrando es aminorar valor al contenido constitucional de la presunción de inocencia.

Existen argumentos que señalan que dado que algunos delitos son cometidos mayormente en la clandestinidad, la única manera para desterrar la violencia de género es otorgando a la víctima un trato de testigo privilegiado, porque de lo contrario estaríamos incumpliendo los compromisos internacionales. Ello deriva en una falacia, pues ningún convenio internacional exige realizar estándares probatorios diferenciados.

El Estado peruano promulgó la Resolución Legislativa n. ${ }^{\circ} 23432$ que aprueba la «Convención sobre la eliminación de todas las formas de discriminación contra la mujer» —en adelante, CEDAWel 5 de junio de 1982; con ello se comprometió a garantizar su cumplimiento efectivo.

Uno de los casos más relevantes resueltos por el CEDAW fue el caso Karen Tayag Vertido vs. Filipinas (2010). Se trató de una mujer cuya ocupación era ser directora ejecutiva en una cámara de comercio, que fue víctima de violación sexual por parte del presidente. Durante el juicio ante las autoridades nacionales, la jueza absolvió al acusado al cuestionar la credibilidad del testimonio de la señora Tayag. Esta última, en la comunicación ante el CEDAW, alegó sufrir una revictimización por el Estado en la medida que no cumplió con su obligación de asegurar que las mujeres estuvieran protegidas contra la discriminación por las autoridades públicas, incluyendo las judiciales. Visto el caso ante el CEDAW, este falló 
a favor de la señora Tayag, razonando que la jueza del caso negó credibilidad a su testimonio en función de cómo debería haber reaccionado antes, durante y después de la violación.

Otro caso relevante en la materia - quizá el más importante en materia de género- es el de Campo Algodonero vs. México, en el cual surgió la desaparición de una serie de mujeres, que habían sido vinculadas con hechos criminales y que fueron ejecutadas por tal motivo. Sin embargo, del análisis del caso se tiene que la actuación de los operadores de justicia mostró una forma de desprecio y poca empatía con aquellas mujeres (por su condición de tal), lo cual alertó a medios nacionales e internacionales. El caso llegó hasta la Corte Interamericana de Derechos Humanos, que lo consideró como uno de violación de derechos de las mujeres y señaló la necesidad de que los Estados parte adopten medidas de protección hacia estas a efectos de evitar que los casos de violencia se sigan suscitando. La sentencia establece la necesidad de los Estados parte de introducir en el ordenamiento penal y procesal penal una serie de medidas destinadas a la investigación y el tratamiento de procesos en materia de género.

Queda claro que muchas veces la valoración de los hechos se encuentra influenciada por estereotipos acerca de lo que se espera de una víctima ideal y racional; sin embargo, nada de ello quiere decir que se deba creer a cabalidad a una supuesta víctima de violencia de género (sea cual sea el delito), y que el hecho de resolver con perspectiva de género - valorar su declaración sin introducir prejuicios que conlleven cualquier tipo de subjetividad- no implica que se invierta la carga de la prueba. No podemos considerar como probado un hecho mientras no exista una corroboración de la declaración única; lo que se pretende concluir es que no amparamos una condena sobre todas las cosas, esto es, a través de la absoluta creencia de la presunta víctima. 
Refiriéndonos al derecho fundamental a la presunción de inocencia, en el ámbito del derecho penal, no se vería justificada su adulteración en un escenario no corroborado, pues estaríamos en un gran retroceso en lo que a los derechos humanos respecta. De ahí que coincidimos con la posición del profesor Pizarro (2019) cuando señala que

Estos objetivos no pueden alcanzarse a través de la creación de un modelo procesal excepcional de carácter cuasi inquisitorial, en el que se prescinda de la inmediación y de la contradicción, o se impida a la defensa el acceso directo a las fuentes de prueba, con las precauciones que se estimen procedentes, desequilibrando con ello la balanza del proceso en favor de la acusación [...] La Justicia penal no puede obtenerse a cualquier precio, y por relevante que sea el bien jurídico que pretenda tutelarse, en ningún caso puede justificar prescindir de la garantía fundamental del derecho de defensa, que constituyen las bases esenciales de nuestro sistema jurídico (p. 22).

\section{CONCLUSIONES}

Considero que si bien la Corte Suprema ha desplegado sus mayores esfuerzos para manejar los casos en los que solo se cuenta con el testimonio de la víctima como medio de prueba, el problema radica en aceptar que el patriarcado aún sigue vigente en nuestro país (aunque en la actualidad se encuentre en retroceso, el derecho fundamental a vivir sin violencia de género no está aun plenamente instaurado, sino que continúa siendo un derecho en progresiva construcción). Por ello es necesario construir un proceso penal con perspectiva de género, esto es, evitar dictar una resolución judicial basada en estereotipos de "víctimas idóneas»; se tiene que llevar a la práctica tal obligación de combatirlos y, a su vez, dejar de optar por la vertiente del punitivismo como doctrina. 
En ese mismo sentido, estimo que existe una evolución legislativa muy apresurada en cuanto a leyes que protejan a la mujer, que debilitan los derechos que le asisten al imputado, producto de la presión de diversos medios de comunicación, discursos políticos - comúnmente populistas - y la falta de satisfacción de los ciudadanos que reclaman mayores penas y disminución de garantías para delincuentes procesados especialmente por delitos basados en género. A donde quiero llegar es que el proceso penal, a mi modesto entender, se creó para proteger a la persona investigada del ius puniendi del Estado, que solo los verdaderos culpables deben verse afectados en sus derechos fundamentales, jamás los inocentes; y mencionamos «verdadero culpable» porque existen falsos culpables, producto de condenas erróneas, ello genera impunidad a los verdaderos ofensores.

Por todo ello, como se explicó en la introducción del presente trabajo, mientras mayores sean las penas, mayor debería ser el análisis de los distintos medios de prueba que se tengan; más aún si solo se cuenta con el testimonio de la víctima, es exigible una corroboración de tal afirmación, y no una corroboración mínima, como afirman algunos autores. Los tres criterios desarrollados en el Acuerdo Plenario n. ${ }^{\circ}$ 02-2005 ya no son suficientes, hay muchos más elementos que uno debería analizar, pero siempre atendiendo al principio de proporcionalidad. Por ejemplo, como se ha mencionado, existen muchos casos en los que la víctima denuncia tardíamente, lo cual dificulta aún más poder arribar a la verdad, al alejarnos de la fecha en que se produjeron los hechos. Claro está que no podemos restar credibilidad a la declaración de la presunta víctima por tal motivo, pues estaríamos haciendo uso de un prejuicio de género. Ya la «Convención sobre la eliminación de todas las formas de discriminación contra la mujer» (CEDAW) ha señalado que no se pueden esperar víctimas ideales y racionales en todos los casos. Sin embargo, a pesar de que en nuestro país cumplimos 
con criterios de valoración probatoria incluso en casos donde solo contamos con la declaración única (a modo de evitar cualquier tipo de estereotipo de género), se intenta asegurar una sentencia condenatoria cuando una causa de este tipo ingresa al sistema de justicia. La propia CEDAW ha señalado que no se exige a los Estados partes realizar estándares probatorios diferenciados. Debe quedar claro que el derecho penal no tiene una vocación de solución de los cimientos de la violencia de género.

Es importante que en tiempos de un presuroso populismo penal, se imponga la racionalidad en cuestiones probatorias. "No se puede castigar a un ciudadano solo porque ello corresponda a la voluntad o a los intereses de la mayoría. Ninguna mayoría, por muy aplastante que sea, puede legitimar la condena de un inocente o la absolución de un culpable» (Ibáñez, 2015, p. 310). Debemos dejar de lado cualquier práctica jurisprudencial que excluya la racionalización del proceso penal, que muchas veces promueve la arbitrariedad y la toma de decisiones meramente subjetivas desnaturalizando los criterios de valoración probatoria.

\section{REFERENCIAS}

Castillo Alva, J. L. (2020). Las garantías mínimas del debido proceso. Iustitia.

Castillo Aparicio, J. (2019). La prueba en el delito de violencia contra la mujer y el grupo familiar. Editores del Centro.

Corte Suprema de Justicia de la República (2005). Pleno Jurisdiccional de las Salas Penales Permanente y Transitoria de la Corte Suprema de Justicia. Acuerdo Plenario n. ${ }^{\circ} 2-2005 / C J-116$. Lima: 30 de septiembre de 2005. 
Corte Suprema de Justicia de la República (2011). VII Pleno Jurisdiccional de las Salas Penales Permanente y Transitoria. Acuerdo Plenario n. ${ }^{\circ}$ 1-2011/CJ-116. Lima: 6 de diciembre de 2011.

Corte Suprema de Justicia de la República (2020). Recurso de nulidad n. ${ }^{\circ}$ 398-2020 Lima Norte. Sala Penal Permanente. Lima: 8 de septiembre de 2020 .

Díaz, S. E. (2019) La prueba de la intención ante casos difíciles y la debida motivación de las decisiones judiciales en el proceso penal peruano [Tesis de licenciatura]. Universidad Privada Antenor Orrego.

Higa, C. (2019). Litigación, argumentación y teoría del caso. Grijley.

Lousada, J. F. (2020). El enjuiciamiento de género. Dykinson.

Mantilla, J. (2016). Derecho y perspectiva de género: un encuentro necesario. Vox Juris, 32(2), 117-125.

Mora, J. J. (2020). Prueba, verdad y razonamiento probatorio. Editores del Centro.

Nieva, J. (2010). La valoración de la prueba. Marcial Pons.

Nieva, J. (2016a). La razón de ser de la presunción de inocencia. InDret. Revista para el Análisis del Derecho, (1), 1-23. https:// indret.com/wp-content/themes/indret/pdf/1203_es.pdf

Nieva, J. (2016b, 30 de junio). La razón de ser de la presunción de inocencia [Video]. En Secretaría Técnica CEI CPP-Perú. https://www.youtube.com/watch? v=dPFxfRgus8Y\&t=134s

Pérez, A. I. (2007). La seguridad como fundamento de la deriva del derecho penal postmoderno. Iustel.

Pizarro, M. (2019). La prueba en los delitos sexuales. Iustitia.

Poder Judicial del Perú (2018). Expediente n. ${ }^{\circ}$ 00199-2018-0-2601JR-PE-01. Primer Juzgado de Investigación Preparatoria. Distrito Judicial de Tumbes: 3 de septiembre de 2018. 
Poder Judicial del Perú (2019). X Pleno Jurisdiccional de las Salas Penales Permanente y Transitorias, III Pleno Jurisdiccional Extraordinario de las Salas Penales Permanente y Transitorias y I Pleno Casatorio Penal. Fondo Editorial del Poder Judicial.

Ramírez, J. L. (2020). El testimonio único de la víctima en el proceso penal desde la perspectiva de género. Quaestio Facti. Revista Internacional sobre Razonamiento Probatorio, (1), 201-246.

Reátegui Sánchez J. y Reátegui Lozano, R. (2017). El delito de feminicidio en la doctrina y en la jurisprudencia. Iustitia. 